\author{
Roberto Márcio de Andrade \\ rma@demec.ufmg.br \\ Universidade Federal de Minas Gerais - UFMG \\ Departamento de Engenharia Mecânica \\ 31270-901 Belo Horizonte, MG, Brasil \\ Alexandre Carlos Eduardo \\ alexandrecarloseduardo@ufsj.edu.br \\ Universidade Federal de São João Del Rei - UFSJ \\ Departamento de Engenharia Mecânica \\ 36307-352 São João Del Rei, MG, Brasil
}

\section{Methodology for Automatic Process of the Fired Ceramic Tile's Internal Defect Using IR Images and Artificial Neural Network}

In the ceramic industry, rarely testing systems were employed to on-line detect the presence of defects in ceramic tiles. This paper is concerned with the problem of automatic inspection of ceramic tiles using Infrared Images and Artificial Neural Network (ANN). The performance of the technique has been evaluated theoretically and experimentally from laboratory and on line tile samples. It has been performed system for IR image processing and, utilizing an Artificial Neural Network (ANN), detecting defected or no defected tile. The system has been applied to detect on-line measurement results achieved at the exit of the press. The above automatic inspection procedures have been implemented and tested on a number of tiles using synthetic and real defects. The results obtained confirmed the efficiency of the methodology defect detection in raw tile and its relevance as a promising approach online, as well as included in quality control and inspection programs.

Keywords: ceramic tiles, defect detection, infrared images, neural network

\section{Introduction}

Ceramic tile is one of the most ancient building materials, numbering thousand-year history, and until now does not get out of fashion, and continues to be improved from year to year. In accordance with the growth in tile demand, there have been some shifts in the world ceramic tile industry. In 1990, Europe accounted for $54 \%$ of world ceramic tile production. By 2002, Europe was down to $25 \%$ of world ceramic tile production. In 2005, world ceramic tile production was about 7 billion sq. meters, of which China produced $35 \%$, and Spain, Italy, and Brazil shared the next $25 \%$, with their production spaced neck and neck (Sezzi, 2006).

Although tile production has increasingly been subject to the introduction of automated technology, with high speed lines now approaching production rates in the order of $200 \mathrm{tiles} / \mathrm{min}$, the control of tile quality, considered fundamental to the maintenance of market share, has often remained essentially a manual operation. Potential, therefore, exists for the increased application of automated inspection, and the effective use of product quality data in the closed-loop control of automated production. The displacement of manual tile inspection procedures through the introduction of automated techniques offers a number of significant commercial and social advantages, including the elimination of human error and/or subjective judgment, improved operational efficiency, and the creation of timely statistical product data, improved safety, better working conditions and reduced labor costs. These important considerations are regarded as fundamental in order to secure a mechanism for competitive improvements within the world tile manufacturing market.

In the production line of ceramic tile industry, several problems can turn into failures of the final product. Among these failures, one of the most important and difficult to monitor is delimitation or void. It manifests itself through layers of parallel air pockets of the pressed material that retain air. Since delimitation produces stress concentrations with high propagation of failures, timely detection of cracks in the structure may help in preventing such failures. Tiles are normally subject to a complete sorting operation at the end of the production cycle, in order to identify all defects, (Zanoni, 1999), or at the exit of kiln, exclusively for identifying the superficial defects (Paganelli, 1999).
An automatic control station for the non-contact detection of green tile delamination, based on IR imaging system, was previously developed (De Andrade et al., 1998; De Andrade et al., 1999). A dynamic IR thermographic test usually involves heating of the target and recording of a sequence of IR images of the excited surface: the evolution of the temperature over time for each particular image element (pixel) of the surface is then analyzed to retrieve information about material defects.

The use of infrared thermal images in an automated procedure for defect location and characterization has some drawbacks, because the first step requires the participation of an expert operator, and the time domain analysis is not always successful due to the difficulty in modeling the inversion problem in any real condition.

In this paper, IR thermograph is connected with Artificial Neural Networks (ANN) for automatic process fault detection in ceramic tile. Artificial neural networks can be seen as highly parallel dynamical systems consisting of multiple simple units that can perform transformation by means of their state response to their input information. How the transformation is carried out depends on the Neural Network (NN) model and its way of learning the transformation. Neural network learns by example. In a typical scenario, a neural network is presented iteratively with a set of sample, known as the training set, from which the network can learn the values of its internal parameters.

To overcome this problem has been considered for a different analysis approach in defect characterization. The main reason for this choice is the defect identification of the problem, a kind of application for which neural networks have been extensively used. Network training is performed using a specimen containing a set of defects at known positions. The approach uses ANN's for detection, defined the number of output nodes corresponding to the number of classes present. During training, the output node corresponding to the class of the input vector is kept clamped at state 1 , while the others are clamped to state 0 . During testing, a winner-take-all mechanism causes the input vector to be classified as belonging to that class corresponding to the output node with the highest activation.

In this paper, the authors describe the integrated system HFDS Hybrid Fault-Detection (IR Images processing and Neural Network) for the detection of defects in ceramic tiles. 


\section{Nomenclature}

$C_{p} \quad=$ specific heat capacity, $\mathrm{J} / \mathrm{kg}^{\circ} \mathrm{C}$

$x, y, z=$ space Cartesian coordinates, $m$

$k \quad=$ thermal conductivity, $\mathrm{W} / \mathrm{m}^{\circ} \mathrm{C}$

$T=$ temperature, $T(x, y, z, t),{ }^{\circ} \mathrm{C}$

$\mathrm{Q}=$ pulse of radiant energy, $\mathrm{W} / \mathrm{m}^{3}$

\section{Greek Symbols}

$\rho \quad=$ density, $\mathrm{kg} / \mathrm{m}^{3}$

$\zeta \quad=$ small depth, $m$

$\Delta \mathrm{t} \quad=$ temperature difference, ${ }^{\circ} \mathrm{C}$

$\Delta \mathrm{x} \quad=$ length, $m$

\section{Description Defects Detection in Ceramic Tile}

As well as many other industries, surface inspection and quality classification is an essential stage in tile manufacturing. Due to the high cost of human inspection, speed of the production line, and repetitious nature of the activity, development of a suitable Automatic Defect Detection System (ADDS) would have an impressive impact on the overall performance of a tile production plant. The key point is eventually being at the performance zenith, which guarantees the success in a very competitive market. To be more specific, the advantages of automatic inspection in the tile industry can be listed as:

Further development to the other stages of the production line: It is possible to spread a modular ADDS throughout the production line to gain a more effective and robust quality control process. Fault prediction and correction, and recycling the defective materials before the kiln will be some of the advantages of such an advanced system.

Although several manufacturers have introduced their commercial inspection systems (see Lebrun (2001) for some examples) and also there have been massive investigations on that field across academics and research groups (to name a few: Baldrich et al., 1999; Boukouvalas et al., 1995; Boukouvalas et al., 1997; Costa and Petrou, 2000; Smith and Stamp, 2000; Valiente et al., 2001), it seems that still more efforts and studies are required to achieve high performance, robust and flexible defect detection algorithms and systems. Table 1 illustrates the typical defects for a ceramic tile.

Table 1. Typical defects of ceramic tiles.

\begin{tabular}{|l|l|}
\hline Defect & Characteristics \\
\hline Broken corners and edges & $\begin{array}{l}\text { Physical damages on corners } \\
\text { and edges }\end{array}$ \\
\hline Colour grading & Changes in color shades \\
\hline Cracks & $\begin{array}{l}\text { Thin and long random } \\
\text { physical defects }\end{array}$ \\
\hline Dirt & $\begin{array}{l}\text { Small random particles on } \\
\text { the surface }\end{array}$ \\
\hline Drops & $\begin{array}{l}\text { Include color and water } \\
\text { drops }\end{array}$ \\
\hline Lines & $\begin{array}{l}\text { Wide visible direct lines on } \\
\text { tile surface, mostly result of } \\
\text { production line bars }\end{array}$ \\
\hline Pinholes & Very small holes \\
\hline Textural problems & $\begin{array}{l}\text { Changes in density and shape } \\
\text { of patterns }\end{array}$ \\
\hline
\end{tabular}

Any defect changes the expected texture of the tile and hence can be interpreted as a textural abnormality. Thus, texture analysis is appropriate for normal and abnormal tile discrimination.

\section{Non-Destructive Thermal Evaluation Methods Principle}

In order to understand the NDTE in the direct problem, the related diffusion heat transfer problem must be analyzed. The structure to be considered is a flat plate containing an inclusion flaw, as shown in Fig. 1. The NDTE requires that a thermal pulse of short duration Q, incident on the surface, and the temperature of surface to be monitored as function of the space. The existence of the flaw alters the thermal resistance of the structure within the flawed region, thus resulting in different temperatures $\mathrm{T}$ on the surface of the structure in the unflawed and the flawed region. Mathematical models were presented in literature, but only for a one-dimensional condition (Williams et al., 1980; Boras et al., 1998). The three-dimensional assumption permits a geometrical independent evaluation of the temperatures in the unflawed and the flawed regions. The diagnostic problem can therefore be faced through the evaluation of the surface temperature on the structure directly above the flaw, comparing it with the surface temperature away from the flaw, when the plate surface is subjected to spatiallyuniform step heat flux input.

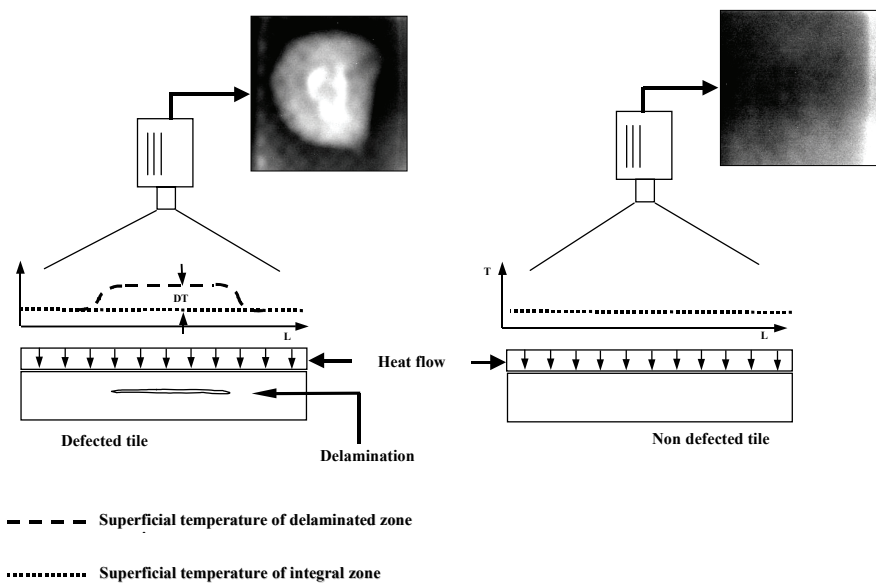

Figure 1. Cross-section of model of plate containing delamination or inclusion flaw.

The basis for theoretical model is differential equation for unsteady heat diffusion in Cartesian coordinates without heat source or sink:

$$
\frac{\partial}{\partial x}\left(k \frac{\partial T}{\partial x}\right)+\frac{\partial}{\partial y}\left(k \frac{\partial T}{\partial y}\right)+\frac{\partial}{\partial z}\left(k \frac{\partial T}{\partial z}\right)+Q=\rho C_{p} \frac{\partial T}{\partial t}
$$

A pulse of radiant energy $Q$ is instantaneously and uniformly absorbed in a small depth $\zeta$ at the surface. Therefore, the initial conditions are:

$$
\begin{aligned}
& T(0,0, z, 0)=\frac{Q}{\rho C_{p} \varsigma}, \text { for } 0<z<\varsigma \\
& T(x, y, z, t)=T_{0}, \text { for } z>\varsigma
\end{aligned}
$$

The boundary conditions are

$$
\left.k \frac{\partial T}{\partial x}\right|_{x=0}=h_{c}\left(T-T_{\infty}\right)
$$




$$
\begin{aligned}
& -\left.k \frac{\partial T}{\partial x}\right|_{x=X L}=h_{c}\left(T-T_{\infty}\right) \\
& \left.k \frac{\partial T}{\partial y}\right|_{y=0}=h_{c}\left(T-T_{\infty}\right) \\
& -\left.k \frac{\partial T}{\partial y}\right|_{y=Y L}=h_{c}\left(T-T_{\infty}\right) \\
& \left.k \frac{\partial T}{\partial z}\right|_{z=0}=h_{c}\left(T-T_{\infty}\right) \\
& -\left.k \frac{\partial T}{\partial z}\right|_{z=Z L}=h_{c}\left(T-T_{\infty}\right)
\end{aligned}
$$

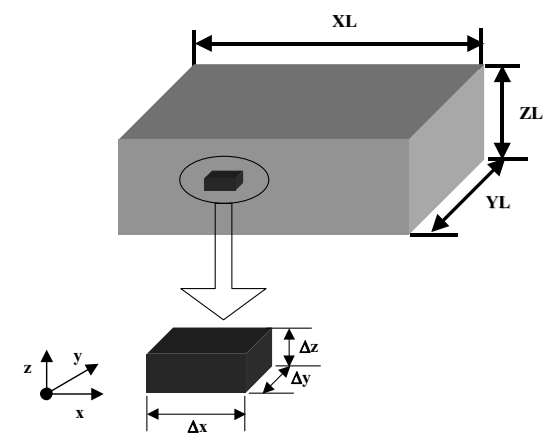

Figure 2. Control volume $P$

The control-volume method, developed by Patankar (1980), has been utilized for the derivation of discretization equation. Then, for each control volume P, Fig. 2, considering fully implicit and piecewise-linear temperature profile between two neighboring grid point (North (N), South (S), East (E), West (W), Top (T), Bot (B)):

$$
\begin{aligned}
& a_{P} T_{P}=a_{E} T_{E}+a_{W} T_{W}+a_{N} T_{N}+a_{S} T_{S}+a_{T} T_{T}+a_{B} T_{B}+a_{P}^{0} T_{P}^{0} \\
& a_{E}=k_{e} \frac{\Delta y \Delta z}{\left(\delta_{x}\right)_{e}} \\
& a_{W}=k_{w} \frac{\Delta y \Delta z}{\left(\delta_{x}\right)_{w}} \\
& a_{N}=k_{n} \frac{\Delta x \Delta z}{\left(\delta_{y}\right)_{n}} \\
& a_{S}=k_{s} \frac{\Delta x \Delta z}{\left(\delta_{y}\right)_{s}} \\
& a_{T}=k_{t} \frac{\Delta x \Delta y}{\left(\delta_{z}\right)_{t}} \\
& a_{B}=k_{b} \frac{\Delta x \Delta y}{\left(\delta_{z}\right)_{b}}
\end{aligned}
$$

$a_{P}^{0}=\frac{\rho C_{p} \Delta x \Delta y \Delta z}{\Delta t}$

$a_{P}=a_{p}^{0}+a_{E}+a_{W}+a_{N}+a_{S}+a_{T}+a_{B}$

A combination of the line-by-line method and a blockcorrection scheme presented by Patankar (1991) is adopted for solution of Eq. 10. The thermal proprieties $K$ and $\rho C_{p}$ have been measured by Flash Method (Parker et al., 1961).

To illustrate the previous theoretical considerations, we present the model considering ceramics of real dimensions $(320 \mathrm{x}$ $320 \times 7 \mathrm{~mm}$ ) that was made with a rectangular defect with dimensions 120 x $120 \times 1 \mathrm{~mm}$, Fig. 3.

The theoretical simulations results are presented in Fig. 4.

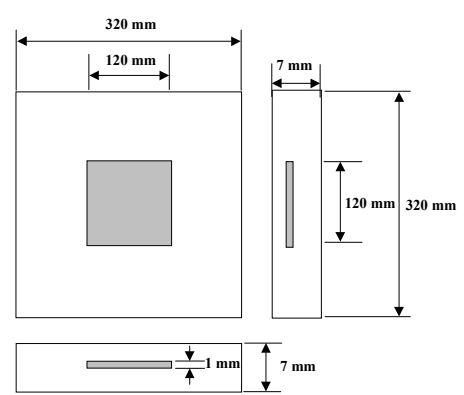

Figure 3. Geometry representation of defected tile for theoretical model.
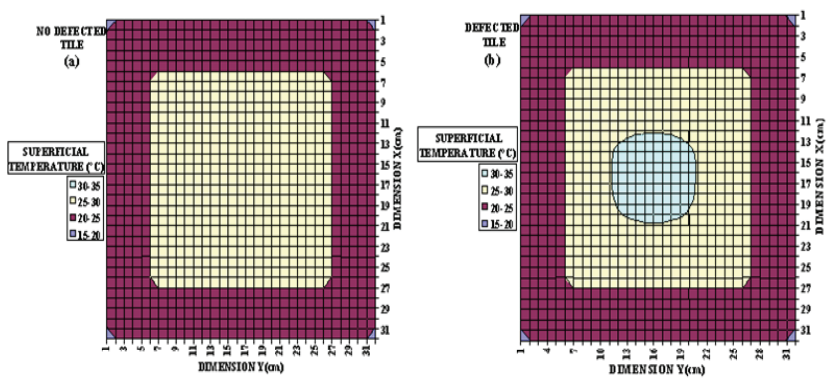

Figure 4. Results of theoretical simulation of NDTE at (a) no defected tile; (b) defected tile.

The presence of the defect generates an increase of the superficial temperature of $5^{\circ} \mathrm{C}$ in the zone of the defect, for the simulated conditions had to the increase of the thermal resistance to the diffusion of heat in the orthogonal direction to the surface.

\section{Artificial Neural Network for Defect Detection}

Detection is the process of sorting pixels into a finite number of individual classes or categories of data based on their original values. If a pixel satisfies a certain set of criteria, then the pixel is assigned to the class that corresponds to that criterion.

The most widely used neural classifier is multilayer perceptron network which has been extensively analyzed and for which many learning algorithms have been developed. The MLP belongs to the class of supervised neural networks. The multi-layer perceptron neural network model consists of a network of processing elements or node arrangement in the layers. Typically, it requires three or more layers of processing nodes: an input layer which accepts the input variables used in the classifier procedure, one or more hidden layers, and an output layer with one node per class. A number of 
neurons in the input layer depend on the features' vector, and in the output layer are based on the number of classes. In this study, a three-layer network has been constructed with 72 neurons in input layer, 40 neurons in hidden layer and 5 neurons in output layer.

There are several training algorithms for feed forward networks. All of these algorithms use the gradient of the performance function to determine how to adjust the weights to optimize performance. The gradient is determined using a technique called back propagation, which involves performing computational backwards through the network.

In this paper, resilient back propagation training algorithm has been used. The algorithm Resilient Back-propagation (RPROP) is a local adaptive learning scheme, performing supervised batch learning in feed-forward neural networks. M. Riedmiller introduced it in 1993. For a detailed discussion see (Riedmiller et al., 1993; Riedmiller, 1994; Riedmiller and Braun, 1993). The basic principle of RPROP is to eliminate the harmful influence of the size of the partial derivative on the weight step. As a consequence, only the sign of the derivative is considered to indicate the direction of the weight update. To achieve this, we introduce for each weight wij(t), its individual update-value $\Delta \mathrm{ij}(\mathrm{t})$, which solely determines the size of the weight-update.

It is introduced a second learning rule, which determines the evolution of the update-value $\Delta_{\mathrm{ij}}(\mathrm{t})$. This estimation is based on the observed behavior of the partial derivative during two successive weight-steps:

$\Delta_{i j}(t)= \begin{cases}\eta^{+} \cdot \Delta_{i j}(t-1), & \text { if } \frac{\partial E}{\partial w_{i j}}(t) \cdot \frac{\partial E}{\partial w_{i j}}(t-1)>0 \\ \eta^{-} \cdot \Delta_{i j}(t-1), & \text { if } \frac{\partial E}{\partial w_{i j}}(t) \cdot \frac{\partial E}{\partial w_{i j}}(t-1)<0 \\ \Delta_{i j}(t-1), & \text { else }\end{cases}$

where

$$
0<\eta^{-}<1<\eta^{+} \text {. }
$$

In words, the adaptation rule works as follows. Every time the partial derivative of the corresponding weight $\mathrm{w}_{\mathrm{ij}}(\mathrm{t})$ changes its sign, which indicates that the last update was too big and the algorithm has jumped over a local minimum, the update-value $\Delta \mathrm{ij}(\mathrm{t})$ is decreased by the factor $\eta-$. If the derivative retains its sign, the update-value is slightly increased in order to accelerate convergence in shallow regions.

Once the update-value for each weight is adapted, the weightupdate itself follows a very simple rule: if the derivative is positive (increasing error), the weight is decreased by its update-value, if the derivative is negative, the update-value is added:

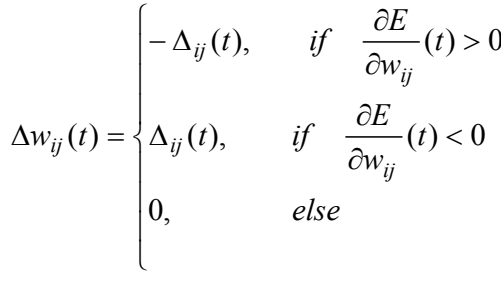

$$
\begin{aligned}
& w_{i j}(t+1)=w_{i j}(t)+\Delta w_{i j}(t) .
\end{aligned}
$$

However, there is one exception. If the partial derivative changes sign, that is, the previous step being too large and the minimum missed, the previous weight-update is reverted:

$$
\begin{aligned}
\Delta w_{i j}(t)= & -\Delta w_{i j}(t-1), \\
& \text { if } \frac{\partial E}{\partial w_{i j}}(t) \cdot \frac{\partial E}{\partial w_{i j}}(t-1)<0
\end{aligned}
$$

Due to that 'backtracking' weight-step, the derivative is supposed to change its sign once again in the following step. In order to avoid a double punishment of the update-value, there should be no adaptation of the update-value in the succeeding step. In practice this can be done by setting $\partial \mathrm{E} / \partial \mathrm{w}_{\mathrm{ij}}(\mathrm{t}-1)=0$ in the $\Delta_{\mathrm{ij}}$ update-rule above.

The partial derivative of the total error is given by

$$
\frac{\partial E}{\partial w_{i j}}(t)=\frac{1}{2} \sum_{p=1}^{P} \frac{\partial E_{p}}{\partial w_{i j}}(t)
$$

Hence, the partial derivatives of the errors must be accumulated for all $\mathrm{P}$ training patterns. This means that the weights are updated only after the presentation of all training patterns. It is a training algorithm that eliminates the harmful effect of having a small slope at the extreme ends of the sigmoid "squashing" transfer functions (Riedmiller, 1993).

\section{Experimental Laboratory}

A laboratory test bench, Fig. 5, has been set in order validation of a theoretical model and to obtain the superficial temperature profile for real tiles from the industrial press. Samples with a dimension of $320 \times 320 \times 7 \mathrm{~mm}$ with known induced defects have been investigated. A sample without defects has been used as a reference. Because of its non-contact nature and full-field measurement capabilities, the IR thermographic technique is a convenient approach to measure the superficial temperature of the sample and for the rapid visualization of shallow surface defects, (Bichard et al., 1976).

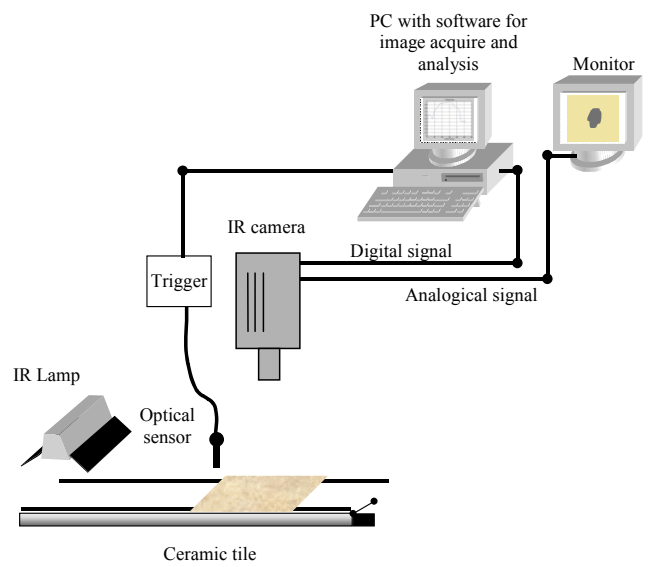

Figure 5. Experimental set-up for infrared thermography experiments.

The superficial thermal energy distribution of tiles has been measured by a Stirling cooled IR camera, with a 256 x $256 \mathrm{CCD}$ array of photo-voltaic InSb detectors working in the 3 to $5 \mu \mathrm{m}$ spectral range (short wave). This system has a Noise Equivalent Temperature Difference (NE $\Delta \mathrm{T}$ ) of about $0.025 \mathrm{~K}$. On line infra red NDTE technique measurements require a fast and precise positioning of the measurement objects, in order to compare spatial distributions of temperature. Therefore, the developed facility has 
been realized with an automatic system for moving, heating and positioning tiles, which simulates the production line. In the tests here presented the samples have been heated with a $2 \mathrm{~kW}$ infra red lamp. The signal from a reflection optical switch, checking the position of the tile, has been used as a trigger for image acquisition.

Figure 6 shows the temperature profiles of experimental simulation of NDTE at no defected and defected tiles. As previewed from theoretical simulation results, the measured temperature difference between the delaminated regions in correspondence with the no defected area is in the order of $5^{\circ} \mathrm{C}$.
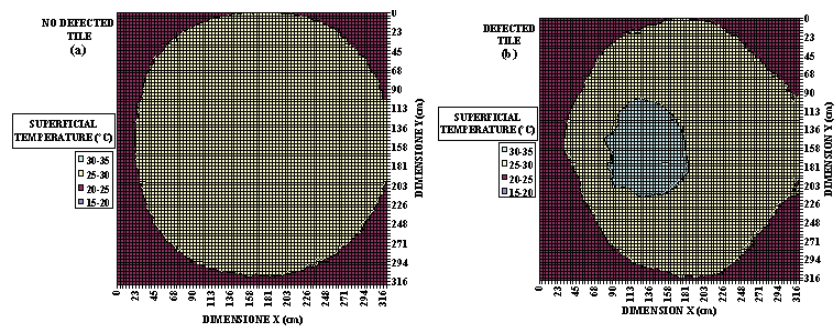

Figure 6. Results of experimental simulation of NDTE at no defected tile (a) and defected tile (b).

\section{Proposed Method}

Once the thermal image has been acquired using the IR camera, a processing procedure must be used in order to generate the input vector for the neural network. This procedure, called "features extraction", aims to extract from the data the features which better highlight the differences between the two classes to be separated and, furthermore, to reduce the amount of data. The procedure here utilized is the following:

The 16 bits grey scale infrared image of the ceramic tile, acquired by the IR camera Radiance ( $256 \times 256$ pixel), is converted into an 8 bits grey image. The contrast of the image is enhanced using image processing software.

The image is integrated along the $\mathrm{Y}$ direction, which is the direction of the tile movement on the production line.

This operation transforms a $256 \times 256$ pixels image in a 256 points line profile. The Y direction has been chosen because, if the line is momentarily stopped, the presence of the rolls under the tile will cause temperature gradients in correspondence with the rolls. Integrating along a direction orthogonal to the rolls, this effect will be reduced and it will just appear in the line profile as a slight increase of the general temperature level, without the introduction of local gradients. On the contrary, if the integration is performed along the rolls' direction (X direction), the temperature gradient due to the rolls would be recognized by the ANN as a defect.

The line profile is normalized between 0 and 1 . It permits to eliminate the dependence of the results from the temperature level, which could vary significantly for the different types of tiles and during the different seasons of the year.

The curve is filtered using a linear digital filter in order to eliminate the noise.

From the 256 points line profile, a 26 points line profile was sampled in such a way as to reduce the amount of data to be processed by the ANN.

\section{Results}

\section{Tests of the Performances of the Automatic Diagnostic System}

Using the curves extracted, the ANN has been trained and tested using images taken both from the lab and on the line at the Leonardo's plant. The results are illustrated in the two following paragraphs. The coefficient of convection, chi, and environment temperature, T $\infty$, has been considered constants. XL, YL and ZL are the dimension of $\mathrm{x}, \mathrm{y}$ and $\mathrm{z}$ direction, respectively, Fig. 2.

\section{Laboratory Tests}

Figure 7 shows the laboratory acquired images and the projection profiles in the $\mathrm{Y}$ direction. The feature, which allows to automatically recognizing the eliminations, is the irregularity of the profiles achieved by processing the images of the defected tiles (see Fig. 7(b)).
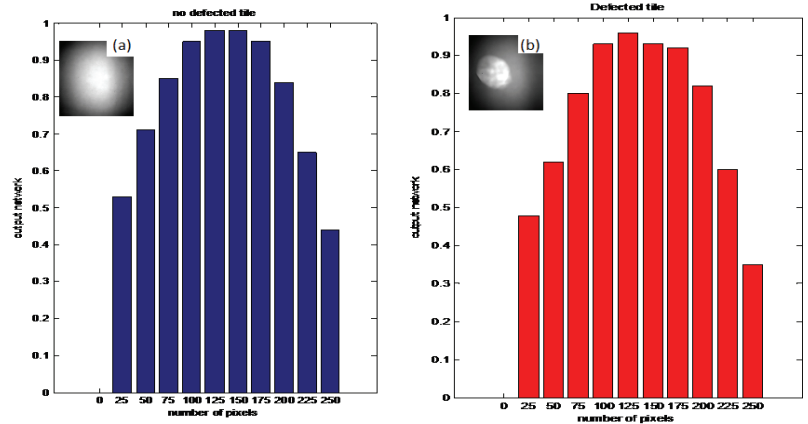

Figure 7. Laboratory IRTI of green tiles: (a) no defected tile, (b) defected tile.

This behavior was found to be extremely repeatable. In these tests a set of 33 samples has been employed for ANN testing. Among these 33 images, 11 have been used as training set and 22 for classification. The classifier system performance was $100 \%$ correct. The laboratory tests have been done in order to optimize the measurement set-up and the ANN architecture before performing the on-line tests.

\section{On-line Tests}

In the press, the temperature of the matrix is higher than the temperature of the powder and, therefore, the tile is heated by the pressing process. This allows performing the IR image acquisition without the application of external thermal pulses. The IR camera has been installed between press and drier, as showed in Fig. 8. A set of 105 images has been acquired with different delaminating typologies.

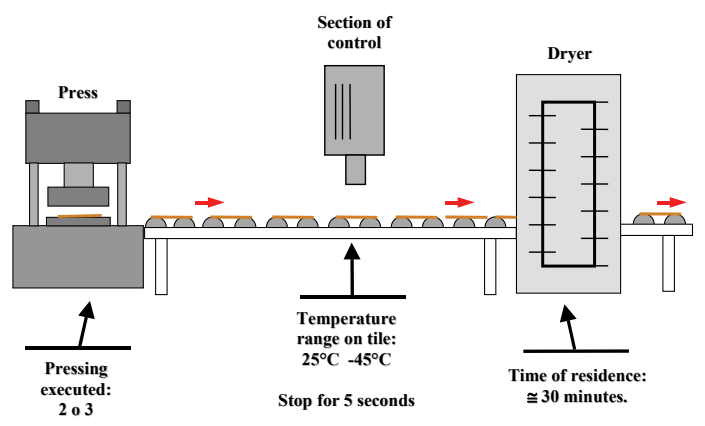

Figure 8. On-line measurement system.

Figure 9 shows examples of results from the on-line tests (the IR thermal images of a no-defected tile (a) and of a defected tile (b) and the projection profiles in the $\mathrm{Y}$ direction of the images). 

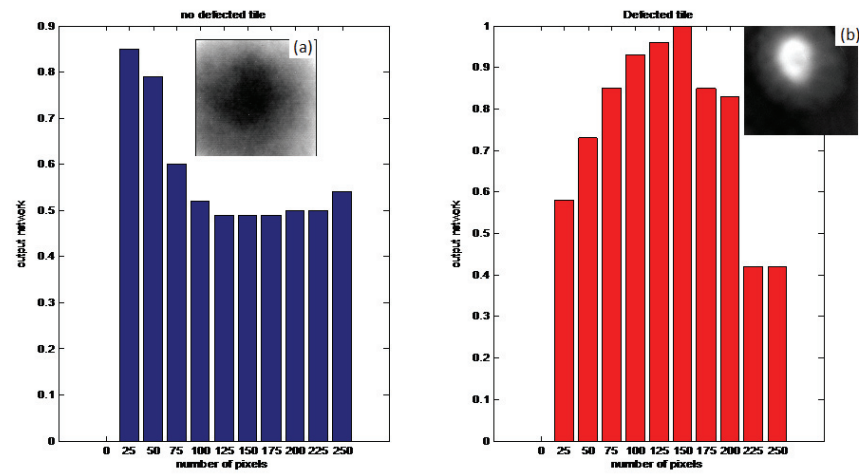

Figure 9. IRTI from on-line tests: (a) no defected tile, (b) defected tile.

In this case, the differences between the two classes seem to be more evident with respect to the laboratory results. Because of the heating procedure from the press matrix, the profiles of the nondefected tile from the on-line tests present higher temperature on the edge and lower in the centre. This behavior (which is opposite from the one observed in the lab, where the tile is heated using the IR lamp) makes the delaminating-induced temperature gradient very well visible. Therefore, the developed feature extraction procedure seems to be appropriate also for on line tests and it is confirmed by the results achieved.

The influence of the temperature level (which can be significantly different in summer and in winter) and the number of pressing executed was eliminated by training a neural network using a training set containing winter and summer thermal images and two and three pressing. 83 images were used for the training set and 22 images for the validation set. In Fig. 10, a result of the feature extraction algorithm is reported.

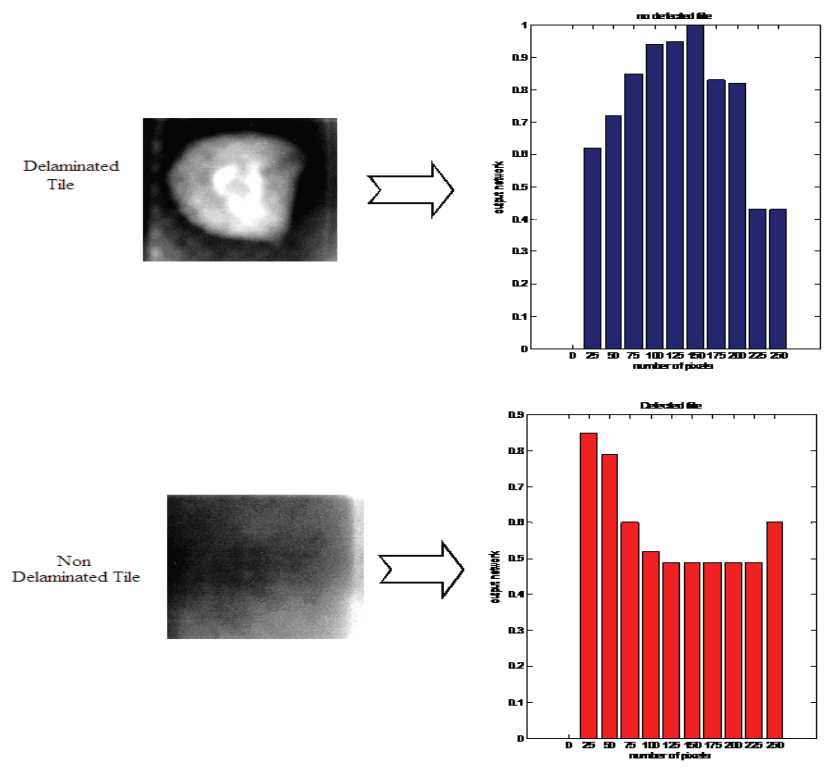

Figure 10. Example of result of the features extraction algorithm from the $256 \times 256$ IR images to the sampled vector.

Figure 11 shows the features of a non-defected tile measured in summer and in winter respectively. Results had proved to be $100 \%$ correct.

The experimental tests show the ability of defect detection in raw tile ceramic in localizing small or large defects in ceramic tiles.
We evaluated our defect detection with $95.87 \%$ sensitivity, $89.47 \%$ specificity, and $92.67 \%$ overall accuracy.
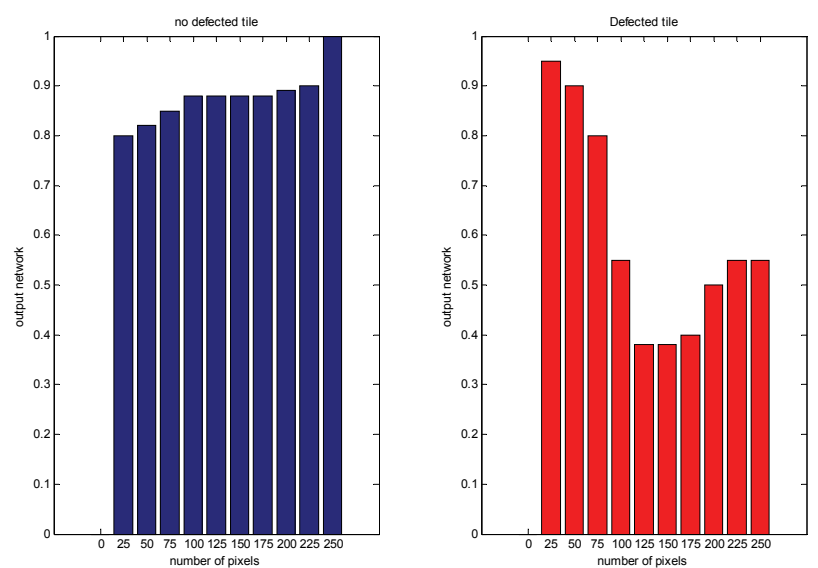

Figure 11. Features extracted from the IR images of a non-defected tile measured in summer and in winter respectively.

\section{Conclusions}

This paper is concerned with the problem of detection of the surface defects included on the fired ceramic tiles using the IR images processing and Artificial Neural Network. The methodology is based on two steps: infrared thermal measurements of the ceramic tile at no defected and defective; processing of the images for generating the input vector for the neural network

By analyzing the theoretical simulation of NDTE at no defected tile and defective tile, it has been concluded that defect generates an increase of the superficial temperature of $5^{\circ} \mathrm{C}$ in the zone on the defect, for the simulate conditions, having to the increase of the thermal resistance to the diffusion of heat in the orthogonal direction to the surface.

The use of Artificial Neural Network for detection defects in ceramic tile is a process of sorting pixels into a finite number of individual classes or categories of data based on their original values. If a pixel satisfies a certain set of criteria, then the pixel is assigned to the class that corresponds to that criterion. In conclusion, the performance of the neural network for detection defects in ceramic tile is very encouraging.

The laboratory acquired images and the projection profiles show irregularities of the profiles achieved by processing the images of the defected tiles.

The on-line measurement system and the differences between the two classes seem to be more evident with respect to the laboratory results. Because of the heating procedure from the press matrix, the profiles of the non-defected tile from the on-line tests present higher temperature on the edge and lower temperature in the centre.

By using this technique, we can develop the sorting system in the ceramic tiles industries from depending on the human which detects the defects manually upon his experience and skills which varies from one to one to the automated system depending on the computer apparatus. Automated sorting systems would bring numerous benefits to the entire sector with major economic advantages, guarantee product quality, increase plant efficiency and reduce fixed and periodic investments. The continuous measurement of surface defects leads line production operators to optimize temperature profile, speed and other operating parameters. 


\section{References}

De Andrade, R.M., Paone, N., Revel, G.M., 1998, "Non Destructive Thermal Detection of Delamination in Ceramic Tile", Proc. ENCIT 98, Rio de Janeiro, pp. 727-731.

De Andrade, R.M., Esposito, E., Paone, N., Revel, G.M., 1999, "Non-destructive Techniques for Detection of Delamination in Ceramic Tiles: a Laboratory Comparison between IR Thermal Cameras and Laser Doppler Vibrometers", In: "Non-destructive Evaluation of Aging Materials and Composites III", G.Y. Baaklini, C.A. Lebowitz, E.S. Boltz, Editors, Proceedings of SPIE, Vol. 3585, pp. 367-377.

Baldrich, R., Vanrell, M., and Villanueva, J., 1999. "Texture-colour features for tile classification", in EUROPTO/SPIE Conference on Colour and Polarisation Techniques in Industrial Inspection.

Bichard, S.H. and Rogers, L.M., 1976. "Industrial Thermography How Representative of Plant Condition is The Thermal Image Produced by an Infrared Camera?", British Journal of Non-Destructive Testing, Vol. 18, No. 4, pp. 111-113.

Boras, I., Svaic, S., 1998, "Determination of the Defect Parameters in Specimen by Thermography and Numerical Methods", In: "Nondestructive Evaluation of Materials and Composites II", Steven R. Doctor, Carol A. Lebowitz, George Y. Baaklini, Editors, Proceedings of SPIE, Vol. 3396, pp. 271-281.

Boukouvalas, C., Kittler, J.R., Marik, M., Mirmehdi, and Petrou, M., 1995, "Ceramic tile inspection for colour and structural defects". In Proceedings of Advanced Materials and Processing Technologies, pp. 390-399, August.

Boukouvalas, C., Vernazza, G., De Natale, F., De Toni, G., Kittler, J., Marik, R., Mirmehdi, M., Petrou, M., Le Roy, P. and Salgari, R., 1997, “An integrated system for quality inspection of tiles". In Proceedings of the International Conference on Quality Control by Artificial Vision, pp. 49-54.

Costa, C., and Petrou, M., 2000, "Automatic registration of ceramic tiles for the purpose of fault detection", Machine Vision and Applications, 11:225-230.

Sezzi, G. , 2006, "World Production and Consumption of Ceramic Tile", Ceramic World Review, Vol. 14, No. 58, pp. 54-71.

Lebrun, V., 2001, "Quality control of ceramic tiles by machine vision", Asian Ceramics: Ceramics manufacturing equipment guide special publication, Retrieved 1 June 2004 from the World Wide Web: http://www.ulg.ac.be/mica/marble/page12.html.

Riedmiller, M., 1993, "Untersuchungen $\mathrm{zu}$ Konvergen und Generalisierungs-verhalen überwachter Lernverfahren mit dem SNNS", in A. Zell, editor, SNNS 1999 Workshop Proceedings, Stuttgart, September.

Riedmiller, M., 1994, "Advanced supervised learning in multi-layer perceptrons-from Backpropagation to adaptive learning algorithms", International Journal on Computer Standards and Interfaces, Vol. 16, pp. $265-278$.

Riedmiller, M., 1994. "Rprop-description and implementation details", Technical Report, University of Kalsruhe, January.

Riedmiller, M., Braun, H., 1993, “A direct adaptive method for faster backpropagation learning: The RPROP algorithm, in H. Ruspini, editor, Proceedings of the IEEE International Conference on Neural Networks (ICNN), San Francisco, USA, pp. 586-591.

Riedmiller, M., and H. Braun, 1993, "A direct adaptive method for faster backpropagation learning: The RPROP algorith", Proceedings of the IEEE International Conference on Neural Networks, San Francisco.

Smith, M., and Stamp, R., 2000, "Automated inspection of textured ceramic tiles". Computer in Industry, Vol. 43, pp. 73-82.

Paganelli, M., 1999, "Pre-selection at the kiln exit", Ceramic World Review, No. 32, June-July, pp. 124-126.

Parker, W.J., Jenkins, R.J., Butler, C.P., Abbot, G.L., 1961, "Flash Method of Determining Thermal Diffusivity, Heat Capacity and Thermal Conductivity", Journal of Applied Physics, Vol. 32, No. 9, pp. 1679-1684.

Patankar, S.V., 1980, "Numerical Heat Transfer and Fluid Flow", Hemisphere Publishing Coporation, Mc Grow-hill Book Co, Washington, USA.

Patankar, S.V., 1991, "Computation of Conduction and Duct Flow Heat Transfer", Innovative Reserach, Inc, USA, 354 p.

Valiente J., Acebron, F., and Lopez, F., 2001, "A ceramic tile inspection system for detecting corner defects", In Proceedings of the IX Spanish Symposium on Pattern Recognition and Image Analysis, Vol. 2, pp. 213-218,.

Zanoni, D, 1999, "Grater automation of the sorting line", Ceramic World Review, No. 33, August-September, pp. 222-227.

William, J.P., 1980, "One Dimensional Analysis of Thermal Nondestructive Detection of Delamination and Inclusion Flaws", British Journal of Non-Destructive Testing, Vol. 22, pp. 113-118. 